\title{
Predictive Value of Serum Lipid Ratio and Homocysteine Determination in Hypothyroidism Complicated with Coronary Heart Disease
}

\author{
Yuzhi Liu ${ }^{1}$, Zhenjiang Hou ${ }^{1}$, Jingyu Zhang ${ }^{2}$, Jianzhang Hou ${ }^{3}$, Hongsheng Wang ${ }^{4}$ \\ ${ }^{1}$ Institute of Thyroid Diseases, Cangzhou Medical College, Cangzhou, China \\ ${ }^{2}$ Laboratory Diagnostics Division, Hebei Cangzhou Hospital of Integrated Traditional Chinese and Western Medicine, Cangzhou, China \\ ${ }^{3}$ Department of Surgery, Cangzhou People's Hospital, Cangzhou Medical College, Cangzhou, China \\ ${ }^{4}$ Diabetes Department of Endocrinology, Hebei Cangzhou Hospital of Integrated Traditional Chinese and Western Medicine, Cangzhou, \\ China
}

\section{Email address:}

houzhenjiang@sina.com (Zhenjiang Hou)

\section{To cite this article:}

Yuzhi Liu, Zhenjiang Hou, Jingyu Zhang, Jianzhang Hou, Hongsheng Wang. Predictive Value of Serum Lipid Ratio and Homocysteine Determination in Hypothyroidism Complicated with Coronary Heart Disease. Science Journal of Public Health.

Vol. 5, No. 6, 2017, pp. 469-473. doi: 10.11648/j.sjph.20170506.20

Received: November 8, 2017; Accepted: November 20, 2017; Published: December 6, 2017

\begin{abstract}
In recent years, with the improvement of detection technology and medical level, the incidence of hypothyroidism, subclinical hypothyroidism and coronary heart disease was increasing year by year and patients were often associated with significant reduction of high-density lipoprotein cholesterol, increase of total cholesterol and Homocysteine and so on. But it was not clear whether there was a similar change occurs in patients with hypothyroidism complicated with coronary heart disease. The authors chose hospital patients, who were divided into a group of hypothyroidism complicated with coronary heart disease (merging group), a group of hypothyroidism, subclinical hypothyroidism and control group, with 60 cases in each group, to measure the blood lipid and Homocysteine, calculate blood lipid ratio and make analysis. As a result, the authors found that patients with hypothyroidism and merging group serum high-density lipoprotein cholesterol decreased. The levels of total cholesterol, triglyceride, low-density lipoprotein cholesterol, total cholesterol / high-density lipoprotein cholesterol, triglyceride / high-density lipoprotein cholesterol, low-density lipoprotein cholesterol / high-density lipoprotein cholesterol, lipid profile, non-high-density lipoprotein cholesterol and Homocysteine were significantly higher than the control group. And the group of patients with hypothyroidism complicated with coronary heart disease was higher than the hypothyroidism group. It came to the conclusion that dynamic monitoring of blood lipid and its ratio and the change of Homocysteine were important for clinical value in transforming subclinical hypothyroidism into hypothyroidism and predicting patients with hypothyroidism complicated with coronary heart disease.
\end{abstract}

Keywords: Hypothyroidism, Subclinical Hypothyroidism, Hypothyroidism Complicated with Coronary Heart Disease, Blood Lipid Ratio, Homocysteine

\section{Introduction}

Hypothyroidism is a common clinical endocrine disease resulting from the reduction of synthesis and secretion of thyroid hormone or the lack of organization and utilization of thyroid hormone, which results in systemic metabolic reduction syndrome. In recent years, with the improvement of detection and awareness of hypothyroidism, the morbidity and diagnostic rate gradually increase [1]. Subclinical hypothyroidism refers to the increase of serum thyroid stimulating hormone and normality of free thyroxine [2]. Due to without clinical symptoms or mild symptoms, subclinical hypothyroidism easily leads to misdiagnosis. As a result, it must be diagnosed according to serological examination. The 
prevalence rate of subclinical hypothyroidism could reach 5\% to $15 \%$ in the crowd with the gradually growth of the increase of age. A woman, who is over 60 , can be as high as $20 \%$ [3]. It is estimated that about $2 \%$ to $5 \%$ of subclinical hypothyroidism transforms into hypothyroidism each year and the latter is an important factor in causing dyslipidemia. The increase of serum thyroid stimulating hormone of subclinical hypothyroidism patients can result in the increase of the incidence of cardiovascular disease [4], which may be associated with atherosclerosis. The atherosclerosis can lead to increased incidence and mortality of coronary atherosclerotic heart disease. It may be another independent risk factor for coronary atherosclerotic heart disease [5]. Researches have shown that the increase of plasma homocysteine was closely related to the occurrence and development of atherosclerosis and was one of the independent risk factors for coronary atherosclerotic heart disease [6]. Hypothyroidism patients with high homocysteine hyperlipidemia predict a higher incidence of cardiovascular disease, especially closed with the atherosclerosis [7]. At present, the correlation between subclinical hypothyroidism and coronary heart disease has been largely studied at home and abroad [8], but there is little research on hypothyroidism complicated with coronary heart disease. It can provide the basis for the prediction and prevention of hypothyroidism complicated with coronary heart disease by measuring the serum thyroid hormone, blood lipid and homocysteine of hypothyroidism, subclinical hypothyroidism, hypothyroidism complicated with coronary heart disease and control group and calculating blood lipid ratio and making correlational analysis.

\section{Materials and Methods}

\subsection{General Information}

The authors selected patients who came to our hospital to take health examination and endocrine treatment from April 2015 to December 2016 with hypothyroidism complicated with coronary heart disease, hypothyroidism, subclinical hypothyroidism and control and there are 60 cases in each group. The 60 cases of hypothyroidism complicated with coronary heart disease included 16 males and 44 females and the ages were from 20 to 77 . The average mean was (50.52 \pm 13.21); 60 cases of hypothyroidism group included 15 males and 45 females and the age was from 21 to 79 . The average mean was $(48.86 \pm 14.59) ; 60$ cases of subclinical hypothyroidism included 17 males and 43 females and the ages were from 23 to 78 . The average mean was (47.61 \pm $15.32) ; 60$ healthy subjects were in the control group. There were 20 males and 40 females and the ages were from 21 to 81 . The average mean was $(47.71 \pm 12.64)$. There was no statistical significance in sex and age among the four groups $(\chi 2=2.877, \mathrm{P}=0.519$ VS $\chi 2=0.898, \mathrm{P}=0.549)$. It was comparable and all of them excluded the history of liver, kidney disease, hypertension and coronary atherosclerotic heart disease, and did not take an impact on thyroid function and blood lipid drugs nearly three months.

\subsection{Methods}

\subsubsection{Detection Method}

All subjects were fasted more than 12 hours and were took to venous blood $6 \mathrm{ml}$ dispensing 2 copies in the morning 7:30 9:30, and centrifuged serum at $4000 \mathrm{r} / \mathrm{min}$ by using the German Roche Cobas E 601 immunochemical luminescence instrument to test serum thyroid stimulating hormone (Thyroid stimulating hormone, TSH), total amount of triiodothyronine (TT3), Thyroxine Total (TT4), Free Triiodothyronine (FT3), Free Thyroxine (FT4). Reagents are original Roche reagents. HITACHI-020 automatic biochemical analyzer was used to test Three acyl glycerol, TG, total cholesterol, TC, High-density lipoprotein cholesterol, HDL-C, Low-density lipoprotein cholesterol, LDL-C, total cholesterol / High-density lipoprotein cholesterol, Three acyl glycerol / High-density lipoprotein cholesterol, Low-density lipoprotein cholesterol / High-density lipoprotein cholesterol, Lipid comprehensive index, LCI = total cholesterol $\times$ Three acyl glycerol $\times$ Low-density lipoprotein cholesterol / High-density lipoprotein cholesterol, nonHigh-density lipoprotein cholesterol $=$ total cholestero High-density lipoprotein cholesterol by using free glycerol method, oxidase method, antibody blocking method and selecting the protection method. Homocysteine was measured by AXSYM automated rapid immunoassay.

\subsubsection{Diagnostic Criteria the Diagnostic Criteria of Hypothyroidism}

Hypothyroidism has corresponding symptoms or signs and Thyroid stimulating hormone $>4.20 \mathrm{mIU} / \mathrm{L}, \quad \mathrm{FT} 4<12$ $\mathrm{pmol} / \mathrm{Land} /$ or FT3 $<3.10 \mathrm{pmol} / \mathrm{L}$; Subclinical hypothyroidism has no corresponding symptoms or signs and Thyroid stimulating hormone $>4.20 \mathrm{mIU} / \mathrm{L}, \mathrm{FT} 3$ and FT4 are normal; Hypothyroidism complicated with coronary heart disease meets the diagnostic criteria for hypothyroidism, and is confirmed the diagnosis of coronary heart disease through the examination of clinical symptoms, electrocardiogram, cardiac troponin and coronary angiography.

\subsection{Statistical Processing}

SPSS 18.0 statistical software was used for data processing. The measurement data was expressed as mean \pm standard deviation $(\overline{\boldsymbol{x}} \pm \mathrm{s})$. Single factor analysis of variance was used for comparsion. The correlations among thyroid hormones and blood lipid profile and Hcy were analyzed by linear correlation analysis and $\mathrm{P}<0.05$ is different, which has statistically significant.

\section{Results}

\subsection{Comparison of Blood Lipid Profile and Hcy Levels in Each Group}

The levels of serum total cholesterol, Three acyl glycero, Low-density lipoprotein cholesterol and homocysteine in the patients with hypothyroidism and hypothyroidism complicated with coronary heart disease were significantly higher than 
those in the control group ( $\mathrm{P}>0.05)$, and the levels of serum total cholesterol, Low-density lipoprotein cholesterol and homocysteine in the hypothyroidism complicated with coronary heart disease group were significantly higher than those in control group $(\mathrm{P}<0.05)$, but there was no significant difference between subclinical hypothyroidism and control group $(\mathrm{P}>0.05)$; The difference of High-density lipoprotein cholesterol between subclinical hypothyroidism, hypothyroidism and hypothyroidism complicated with coronary heart disease group was lower than that of control group $(\mathrm{P}>0.05)$. There was no significant difference between the latter two groups $(\mathrm{P}>0.05)$. See Table 1.

Table 1. Comparison of blood lipid (mmol / L) and Hcy levels in each group $(\overline{\boldsymbol{x}} \pm S)$.

\begin{tabular}{lllllll}
\hline Groups & $\mathbf{n}$ & TC $(\mathbf{m m o l} / \mathbf{L})$ & TG $(\mathbf{m m o l} / \mathbf{L})$ & HDL-C $(\mathbf{m m o l} / \mathbf{L})$ & $\mathbf{L D L}-\mathbf{C}(\mathbf{m m o l} / \mathbf{L})$ & Hcy $(\boldsymbol{\mu m o l} / \mathbf{L})$ \\
\hline Merging & 60 & $6.241 \pm 1.621^{*}$ & $2.036 \pm 1.52 *$ & $1.171 \pm 0.418^{*}$ & $4.021 \pm 0.94 *$ \\
Hypothyroidism & 60 & $5.441 \pm 1.596^{*}$ & $1.951 \pm 1.58^{*}$ & $1.211 \pm 0.397^{*}$ & $3.681 \pm 1.04 *$ \\
Subclinical Hypothyroidism & 60 & $5.113 \pm 1.128$ & $1.631 \pm 1.149$ & $1.265 \pm 0.326$ & $3.112 \pm 0.887$ \\
Control & 60 & $4.898 \pm 0.894$ & $1.608 \pm 1.179$ & $1.361 \pm 0.331$ & $3.130 \pm 0.369$ & $13.979 \pm 36.889 *$ \\
\hline
\end{tabular}

Note: Compared with the control group, $* \mathrm{P}<0.05, * * \mathrm{P}<0.01$.

\subsection{Comparison of Blood Lipid Ratio, Lipid Comprehensive Index and non-High-Density Lipoprotein Cholesterol}

The levels of Three acyl glycero / High-density lipoprotein cholesterol, Low-density lipoprotein cholesterol / High-density lipoprotein cholesterol, Lipid comprehensive index and nonHigh-density lipoprotein cholesterol in hypothyroidism and hypothyroidism complicated with coronary heart disease group were significantly higher than those in control group. The total cholesterol / High-density lipoprotein cholesterol of hypothyroidism and hypothyroidism complicated with coronary heart disease was also higher than the control group $(\mathrm{P}<0.05)$, but there was no significant difference among the groups $(\mathrm{P}>0.05)$. See Table 2 .

Table 2. Comparison of Blood Lipid Ratio, LCI and Non-HDL-C in Eadministratorch Group.

\begin{tabular}{|c|c|c|c|c|c|c|}
\hline Groups & $\mathbf{n}$ & TC/HDL-C & TG/HDL-C & LDL-C/HDL-C & LCI & non-HDL-C \\
\hline Merging & 60 & $4.031 \pm 1.20^{*}$ & $1.808 \pm 1.29 *$ & $3.859 \pm 0.94 *$ & $36.96 \pm 40.47 * *$ & $4.331 \pm 1.26^{*}$ \\
\hline Hypothyroidism & 60 & $3.942 \pm 1.09 *$ & $1.606 \pm 1.29 *$ & $3.393 \pm 0.86^{*}$ & $33.83 \pm 47.16^{* *}$ & $4.017 \pm 1.39 *$ \\
\hline Subclinical Hypothyroidism & 60 & $3.923 \pm 1.03 *$ & $1.358 \pm 1.26$ & $2.962 \pm 0.779$ & $24.409 \pm 28.106$ & $3.911 \pm 1.08^{*}$ \\
\hline Control & 60 & $3.371 \pm 1.02$ & $1.321 \pm 1.23$ & $2.391 \pm 0.821$ & $23.383 \pm 29.409$ & $3.594 \pm 0.887$ \\
\hline
\end{tabular}

Note: Compared with the control group, $* \mathrm{P}<0.05, * * \mathrm{P}<0.01$.

\section{Discussions}

Thyroid dysfunction has great impact on blood lipid. Dyslipidemia, which is caused by hypothyroidism, has become an important reason for atherosclerosis. A study of 295 cases of primary hypothyroidism shows that $91.5 \%$ of patients have hyperlipidemia, followed by type IIa (simple hypercholesterolemia, 56\%), type IIb (hypercholesterolemia complicated with high triglyceride, accounting for 34\%). About $30 \%$ of patients with hypothyroidism have a great effect on subclinical hypothyroidism and Low-density lipoprotein cholesterol manifested as elevated and they have a significant effect on Three acyl glycerol, High-density lipoprotein cholesterol and Very low-density lipoprotein cholesterol. In the normal range, the level of higher Thyroid stimulating hormone and lower thyroid hormone are associated with lipid along with Thyroid stimulating hormone increased and subclinical hypothyroidism, Low-density lipoprotein cholesterol and Three acyl glycero are positively correlated, and negatively correlated with High-density lipoprotein cholesterol [9]. Qingzhang Song et al [10] reported that the Three acyl glycero, total cholesterol, Low-density lipoprotein cholesterol of patients with hypothyroidism were significantly higher than the control group, and High-density lipoprotein cholesterol was significantly lower than the control group. The slight changes of Thyroid stimulating hormone of subclinical hypothyroidism patients also affected lipid metabolism. Toruner et al found [11] that the total cholesterol, Three acyl glycero and Low-density lipoprotein cholesterol of subclinical hypothyroidism patients regardless of Thyroid stimulating hormone $\geq 10 \mathrm{mIU} / \mathrm{mL}$, or Thyroid stimulating hormone $<10 \mathrm{mIU} / \mathrm{mL}$, were significantly higher than the control group. Van Tienhoven-wind et al [12] reported that serum subclinical hypothyroidism, Three acyl glycero and Low-density lipoprotein cholesterol of subclinical hypothyroidism patients significantly increased. Caihong Liu et al [13] found that subclinical hypothyroidism patients with increasing total cholesterol and decreasing High-density lipoprotein cholesterol. The incidence of coronary atherosclerotic heart disease increased. Wei Liu et al $[14,15]$ reported that Thyroid stimulating hormone, Three acyl glycero, subclinical hypothyroidism, Low-density lipoprotein cholesterol of hypothyroidism complicated with coronary heart disease significantly increased, while High-density lipoprotein cholesterol decreased, and Low-density lipoprotein cholesterol was an independent risk factor for coronary atherosclerotic heart disease in patients with subclinical hypothyroidism. This study found that serum subclinical hypothyroidism, Three acyl glycero, Low-density lipoprotein cholesterol of patients with hypothyroidism were significantly higher than the subclinical hypothyroidism group and control group, and High-density lipoprotein cholesterol decreased. The serum of subclinical 
hypothyroidism and Low-density lipoprotein cholesterol of hypothyroidism complicated with coronary heart disease group were higher than those in hypothyroidism group ( $\mathrm{P}$ $<0.05$ ). The level of High-density lipoprotein cholesterol in subclinical hypothyroidism, hypothyroidism and hypothyroidism complicated with coronary heart disease were lower than that in the control group. And the results were basically the same as the above research. Studies have confirmed that lipid metabolic disorder of hypothyroidism increase the risk of morbidity of coronary atherosclerotic heart disease. Therefore, correction of hypothyroidism in patients with lipid metabolism disorders was of great significance for preventing hypothyroidism complicated with coronary heart disease.

Data indicated that the combination of high Three acyl glycero, subclinical hypothyroidism and low High-density lipoprotein cholesterol had greater risk of coronary atherosclerotic heart disease than single dyslipidemia. The ratio of Three acyl glycero and total cholesterol / High-density lipoprotein cholesterol was good indicators for assessing coronary atherosclerotic heart disease risk. Subclinical hypothyroidism / High-density lipoprotein cholesterol was stronger than single-use subclinical hypothyroidism, Low-density lipoprotein cholesterol and High-density lipoprotein cholesterol in predicting the risk of coronary artery and was higher than that of single lipid. Subclinical hypothyroidism / High-density lipoprotein cholesterol, Low-density lipoprotein cholesterol / High-density lipoprotein cholesterol predicted a significant increase in the risk of myocardial infarction. Therefore, subclinical hypothyroidism / High-density lipoprotein cholesterol, Low-density lipoprotein cholesterol / High-density lipoprotein cholesterol and other blood lipid ratio were more valuable than a single indicator of the diagnosis of coronary atherosclerotic heart disease [18]. The prediction of Three acyl glycero / High-density lipoprotein cholesterol ratio was more effective in the incidence of coronary atherosclerotic heart disease and atherosclerosis and was a reliable indicator of coronary atherosclerotic heart disease and coronary artery disease. Lipid comprehensive index $=$ subclinical hypothyroidism $\times$ Three acyl glycero $\times$ Low-density lipoprotein cholesterol / High-density lipoprotein cholesterol could reflect the lipid abnormalities and coronary atherosclerotic heart disease. What's more, its sensitivity was higher than any single lipid index. Moreover, it was more valuable in the risk of coronary atherosclerotic heart disease, monitoring the severity of the disease and prognosis. Yinjun Xie [16] reported that Lipid comprehensive index of coronary atherosclerotic heart disease group was significantly higher than the control group. It was a good indicator of the risk of assessing coronary atherosclerotic heart disease. The prognosis of monitoring coronary atherosclerotic heart disease also had some value. Non- High-density lipoprotein cholesterol represented the total amount of atherosclerotic cholesterol, and its predictive value of coronary atherosclerotic heart disease was superior to the traditional lipid index. The results of this study showed that Three acyl glycero / High-density lipoprotein cholesterol, Lipid comprehensive index and non- High-density lipoprotein cholesterol were significantly higher in patients with hypothyroidism than in subclinical hypothyroidism group and control group $(\mathrm{P}<0.05)$. Low-density lipoprotein cholesterol / High-density lipoprotein cholesterol of subclinical hypothyroidism group was significantly higher than the hypothyroidism group and control group; The levels of Three acyl glycero / High-density lipoprotein cholesterol, Low-density lipoprotein cholesterol / High-density lipoprotein cholesterol, Lipid comprehensive index and non- High-density lipoprotein cholesterol in the hypothyroidism and hypothyroidism complicated with coronary heart disease group were significantly higher than those in the control group. The levels of subclinical hypothyroidism / High-density lipoprotein cholesterol in subclinical hypothyroidism, hypothyroidism and hypothyroidism complicated with coronary heart disease were also higher than those in control group $(\mathrm{P}<0.05)$. The correlation between various thyroid hormone and lipid ratio and non- High-density lipoprotein cholesterol was the highest correlated with subclinical hypothyroidism, followed by nonHigh-density lipoprotein cholesterol, Low-density lipoprotein cholesterol and Lipid comprehensive index, respectively [17]. Blood lipid measurement had become a routine project of Cardiology and calculating Three acyl glycero / High-density lipoprotein cholesterol, Lipid comprehensive index and nonHigh-density lipoprotein cholesterol was convenient, free from dietary and gender effects, which has opened up a new way for the prediction of hypothyroidism complicated with coronary heart disease, and had wide application prospect.

High homocysteine hyperthyroidism can increase the incidence of coronary atherosclerotic heart disease and homocysteine increased by $4 \mu \mathrm{mol} / \mathrm{L}$ coronary atherosclerotic heart disease can increase the risk of $4 \%$, and homocysteine increased $5 \mu \mathrm{mol} / \mathrm{L}$ and total cholestero increased by 0.5 mmol / L to the same extent to increase the incidence of coronary atherosclerotic heart disease [18]. Qingzhang Song et al [10] considered that the increase of serum homocysteine was an important risk factor for hypothyroidism complicated with coronary atherosclerotic heart disease. The determination of serum Hcy level was of great value in the diagnosis, treatment and prognosis of hypothyroidism complicated with coronary atherosclerotic heart disease. Chunjuan Zhou et al [7] found that in the process of transformation from subclinical hypothyroidism to hypothyroidism, the change of homocysteine was positively correlated with Thyroid stimulating hormone. The increase of serum homocysteine who were hypothyroidism patients indicated a higher incidence of cardiovascular disease. Combing detection of serum Thyroid stimulating hormone with homocysteine was of great value on the diagnosis and treatment of hypothyroidism. Lu Xiong et al [19] stratified study found that a slight increase in Thyroid stimulating hormone levels $(<10 \mathrm{mIU} / \mathrm{L})$ was not sufficient to affect Three acyl glycero and Low-density lipoprotein cholesterol, but the level of plasma homocysteine increased $(\mathrm{P}<0.05)$. The significant increase of Thyroid stimulating hormone ( $\geq 10 \mathrm{mIU} / \mathrm{L})$ could let Three acyl glycero, Low-density lipoprotein cholesterol and homocysteine 
levels significantly increase $(\mathrm{P}<0.05)$. This study found that serum homocysteine of subclinical hypothyroidism, hypothyroidism and hypothyroidism complicated with coronary heart disease were significantly higher than the control group $(\mathrm{P}>0.05)$. The difference among the four groups was statistically significant and consistent with the above findings. It is suggested that with the occurrence of subclinical hypothyroidism, hypothyroidism and hypothyroidism complicated with coronary heart disease, serum homocysteine levels gradually increased. Therefore, dynamic monitoring change of homocysteine contributes to prediction of transformation from subclinical hypothyroidism to hypothyroidism and hypothyroidism complicated with coronary heart disease. However, the authors have not yet conducted a large sample of prospective studies.

\section{Conclusions}

In short, the patients of hypothyroidism, subclinical hypothyroidism and hypothyroidism complicated with coronary heart disease have significant lipid metabolism disorders and increase of Homocysteine. Dynamic monitoring of Thyroid stimulating hormone, lipid ratio, Homocysteine and cardiac function has important clinical value in the prediction of transformation from subclinical hypothyroidism to hypothyroidism and hypothyroidism complicated with coronary heart disease and other cardiovascular diseases, developing a reasonable treatment program and improving and promoting the efficacy and prognosis of hypothyroidism and hypothyroidism complicated with coronary heart disease and other cardiovascular disease.

\section{Acknowledgements}

This study was supported by a project grant from: The Science and Technology Plan Project in Hebei Province (Grant No. 15277796D).

\section{References}

[1] Chu XQ, Liu C. New thoughts on pathological factors of hypothyroidism. Chin J Prac Inter Med, 2014, vol. 34, pp. 313-316.

[2] Bensenor IM, Olmos RD, Lotufo PA. Hypothyroidism in the elderly: diagnosis and manage ment. Clin Interv Aging, 2012, vol. 7, pp.97-111.

[3] Lioudaki E, Mavroeidi NG, Mikhailidis DP, et al. Subclinical hypothyroidism and vascular risk:an update. Hormones (Athens), 2013, vol. 12, pp.495-506.

[4] Kamasaki H, Takeuchi T, Mikami T, et al. A case of graves' disease diagnosed in the course of bilateral carotid artery stenoses (moyamoya disease ); a case report and review of the literature. Clin Pediatr Endocrinol, 2013, vol. 22, pp. 39-44.
[5] Rotondi M, Magri F, Chiovato L. Risk of coronary heart disease and mortality for adults with subclinical hypothy-roidism.JAMA, 2010, vol. 304, pp. 2481.

[6] Schaffer A, Verdoia M, Cassetti E, et al. Relationship between homocysteine and coronary disease, Results from a large prospective cohort study. Thromb Res, 2014, vol. 134, pp. 288-293.

[7] Zhou CJ, Zhu XL, Wang CG, et al.. The diagnosis value of combined detection of $\mathrm{TH}$ and Hcy levels for patients with hypothyroidism. Chin J Health Lab Tec, 2016, vol. 26, pp. 2205-2207.

[8] Tseng FY, Lin WY, Lin CC, et al. Subclinical hypothyroidism is associated with increased risk for all-cause and cardiovascular mortality in adults. J Am Coll Cardiol, 2012, vol.60, pp. 730-737.

[9] Fu YL, Fan LM. Research progress on the correlation between thyroid function and metabolic syndrome. J Mod Med Health, 2017, vol. 33, pp.77-79.

[10] Song QZ, Xu FL, Bi XP, et al. Clinical significance of serum hom ocysteine and lipids determ ination in hypothyroi-dism. Lab Med, 2007, vol. 22, pp. 176-178.

[11] Toruner F, Altinova AE, Karakoc A, et al. Risk factorsfor cardiovascular disease in patients with subclinical hypothyroidism. Adv Ther, 2008, vol. 25, pp. 430-437.

[12] van Tienhoven-Wind LJ, Dullaart RP. Low-normal thyroid function and the pathogenesis of commoncardio-metabolic disorders. EurJClin Invest, 2015, vol. 45, pp. 494-503.

[13] Liu CH, Xiao JX. To investigate the correlation between subclinical hypothyroidism and coronary heart disease. Prac Clin J Inte Tradi Chin Wes Med, 2016, vol. 16, pp.40-41.

[14] Liu W, Pan M, Wu XH. Effect of subclinical hypothyroidism on metabolism factors in elderly patients with coronary heart disease. Chin J Clin Health, 2015, vol. 18, pp. 354-356.

[15] Wang C, Zhang P. The clinical study of blood lipid ratio in predicting cardiovascular diseaseProgress. Shandong J Med, 2015, vol. 36, pp. 99-102.

[16] Xie YJ. The level and significance of serum lipid index in patients with different types of coronary heart disease. Contemporary Med, 2012, vol. 18, pp. 10-11.

[17] Hou Zhenjiang, Mu Zhaoxin, Zhang Jingyu, et al. The Correlation of Blood Lipid Profile and its Ratio, Cystatin C and Homocysteine of Thyroid Dysfunction. American Journal of Clinical and Experimental Medicine, 2017, vol. 5, pp. $108-114$

[18] Wu ZR, Zhang LR, Pan YQ et al. Changes and clinical significance of serum homocysteine and lipids in Patients with primary hypothyroidism and hyperthyroid. Prog Mod Bio, 2009, vol. 22, pp. 4276-4278.

[19] Xiong L, Luo CD, Liu YB, et al. Changes of Plasma Homocysteine and Peripheric Arterial Stiffness in Patients with Subclinical Hypothyroidism. West Chin J Med, 2015, vol. 30, pp. $2205-2207$. 